\title{
Erratum to: Modelling spatially explicit impacts from phosphorus emissions in agriculture
}

\author{
Laura Scherer $^{1} \cdot$ Stephan Pfister ${ }^{1}$
}

Published online: 25 June 2015

(C) Springer-Verlag Berlin Heidelberg 2015

Erratum to: Int J Life Cycle Assess (2015) 20:785-795

DOI 10.1007/s11367-015-0880-0

In the published version of this article, there was an error in the electronic supplementary material.

There was a shift in the rows in the excel file "ESM2.xls" provided as electronic supplementary material so that data rows and row names did not match. The corrected ESM has been uploaded.

The online version of the original article can be found at http://dx.doi.org/ 10.1007/s11367-015-0880-0.

Electronic supplementary material The online version of this article (doi:10.1007/s11367-015-0919-2) contains supplementary material, which is available to authorized users.

Laura Scherer

scherer@ifu.baug.ethz.ch

Institute of Environmental Engineering, ETH Zurich,

8093 Zurich, Switzerland 